\section{AKADEMOS}

\section{ISSN: 1995-4743}

Año 13 Vol. 1, n³2 Enero-Junio 2019

AKADEMOS es una revista semestral. De amplio espacio editorial, para la publicación de trabajos inéditos de investigación, artículos de análisis, reseñas y opinión, en los distintos tópicos de las ciencias, la tecnología, las artes y la cultura.

\title{
El acceso a la educación superior pública en El Salvador. Una aproximación al problema
}

\section{Mauricio Santiago}

Santa Ana, El Salvador (1990). Licenciado en Ciencias de la Educación, especialidad en primero y segundo ciclo de educación básica. Maestro en profesionalización de la docencia superior. Docente universitario en las carreras de profesorado y licenciatura en ciencias de la educación, y en maestrías de docencia superior.

msantiag05_45@yahoo.com

\section{Resumen}

La educación superior es un servicio educativo cada vez más demandado. Ante el reto de la sociedad cambiante, cada vez más compleja y exigente en la formación profesional, la educación superior aparece como la disciplina que se presta para llevar a cabo la misión de formar a las generaciones en el ámbito profesional. Cada año son más los bachilleres que aspiran a la educación superior, más que todo a la financiada por el Estado salvadoreño; no obstante, las posibilidades de ser admitido en educación superior no son grandes, pues antes el aspirante debe aprobar un examen de admisión, que menos de la mitad de los aspirantes supera.

$¿$ Es, entonces, realmente un derecho el acceso a la educación pública? Sobre esta interrogante gira este escrito. Para responder al problema, se ha consultado una estadística de aspirantes y admitidos desde el año 2014, así como la normativa legal que respalda el derecho a la educación y de teoría, para explicar desde el punto de vista socioeducativo el acceso a la educación superior.

Palabras clave: educación superior, examen de admisión, marco legal, política educativa. 


\section{Abstract}

The higher education is an educative service increasingly demanded. In front of the challenge of the changer society, increasingly complex and exigent in the professional education, the higher education appears as an institution that lends itself to carry out the mission of training the generations in the professional ambit. Every year more student from high school that try to apply to the higher education, that sometimes is financed by the Salvadorian's government, in the others hand, the possibilities to be accepted in these institution is not big, because before they must pass an exam to have admission's right that less of the half of all students surpasses. So, is it a right of access to the public education? This question is the reason that this document was writing. To answer this problem, we have consulted for the statistics of the aspirants and accepted since 2014, like the legal normative that supports the right of education and the reason for explain since that socio-educative point of view access to the higher education.

Key words: higher education, admission exam, legal framework, educational policy.

Todo bachiller sueña con ir a estudios universitarios. Acceder a la Universidad pública es algo que pocos logran. ¿Por qué pocos?
¿Acaso no es un derecho del Estado garantizarla? ¿Qué es lo que evita que todos ingresen a la Universidad? ¿No hay leyes que garantizan el acceso a la educación superior? ¿Hay factores sociales y económicos que determinan las posibilidades de acceder? Estas son algunas de las interrogantes que me motivaron a buscar respuestas, las cuales presento aquí en este breve ensayo, que tampoco es un estudio minucioso, pero sí básico para empezar y fomentar la discusión de dicha problemática.

\section{Situación actual}

En nuestro país la Universidad de El Salvador (UES) es la única institución de índole pública que promueve la educación superior. Su bajo costo económico en escolari$\mathrm{dad}^{1}$, su estatus académico y su larga historia la convierten en prioridad para la mayoría de bachilleres que desean realizar estudios superiores. Sin embargo, no todos tienen la posibilidad de acceder, no precisamente por falta de condiciones económicas y sociales, sino porque también no superan el filtro de admisión que se ejecuta a través de un examen estandarizado; además está determinado por el número de cupos que se designan a cada facultad o carrera específica.

La medida del examen de admisión para ingresar a la UES es un mecanismo que hace frente a un problema mayor en la educación

Según el Art. 56 de la Constitución de la República, la educación gratuita impartida por el Estado solo abarca hasta la educación media (Asamblea Legislativa, 2017), por lo tanto, para la educación superior se deben pagar cuotas económicas de escolaridad. No obstante, debido a los grandes esfuerzos de las autoridades de la UES se ha logrado que en la actualidad aquellos estudiantes que provienen de instituciones educativas públicas están exentos de pagar matrícula y cuotas. 
superior: su falta de financiamiento adecuado y, a la vez, de interés en las políticas educativas gestionadas que doten los recursos necesarios. Esto ha ralentizado su expansión y fortalecimiento de infraestructura para dar cobertura a un número en crecimiento de bachilleres graduados.

Todo lo anterior tiene implicaciones importantes en educación superior, considerando la alta tasa de población en edades para acceder a educación superior, donde según la Encuesta de Hogares con Propósitos Múltiples el $53.6 \%$ de la población es menor de 30 años (Ministerio de Economía, 2017). Este dato dice mucho de la cantidad significativa de estudiantes que pueden ingresar o están en la Universidad.

Aquí entra el primer problema: cómo garantizar las condiciones (presupuestarias, huma- no, infraestructura, recursos tecnológicos, entre otros) necesarias de la Universidad para atender a todos los estudiantes. Un primer panorama está en la infraestructura con que se cuenta, que en comparación a las universidades privadas, se queda demasiado corta.

En cuanto a infraestructura, actualmente la UES cuenta con cuatro centros de estudio: Ciudad Universitaria, Facultad Multidisciplinaria de Occidente, Facultad Multidisciplinaria de Oriente y Facultad Multidisciplinaria Paracentral. No obstante, las cuatro instalaciones no dan abasto a los 55, 291 matriculados para el año 2019; igualmente, en el año 2018, para el ciclo I hubo 55, 795; y para el ciclo II hubo 47,864 estudiantes matriculados (Universidad de El Salvador, Secretaría de asuntos académicos, 2019), cantidad que ha sido constante desde el año 2014, tal como se ve en el siguiente gráfico:

\section{Gráfica 1: Matrícula de estudiantes de la UES. Se presentan los datos de matrícula desde el año 2014 hasta la actualidad.}

\section{Matrícula anual}

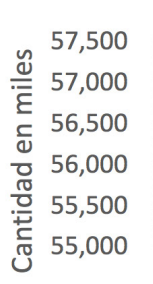

57,323

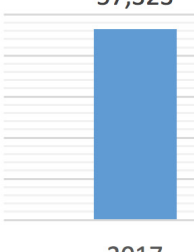

2017

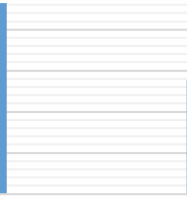

56,390

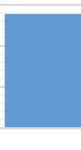

2016

55,850

55,921

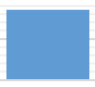

2015

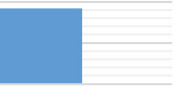

2014

Año

Fuente: Creación propia a través de datos estadísticos de la Universidad de El Salvador (2017). 
Puede observarse que la matrícula se ha mantenido en una constante de 55, 000 como mínimo, distribuidos en los tan solo cuatro centros de estudios ya citados, lo que vuelve a estos centros, espacios sobresaturados, con aulas en algunos casos con más de 100 estudiantes a cargo de un solo docente, lo que no permite que se cumplan los requisitos mínimos pedagógicos ni didácticos. En pocas palabras, las condiciones infraestructurales no están a la altura de la cantidad de estudiantes que atiende.

Si se compara la matrícula de la Universidad pública con las privadas (hay 23 a nivel nacional), la diferencia es abismal. Según el MINED (2015, 2016, 2018a, 2018b) la matrícula total de las universidades privadas para el año 2014 fue de 116,355 estudiantes, en 2015 fue de 119,689, para el año 2016 se obtuvo una matrícula de 121,739, y el año 2017 fue 123,100 estudiantes, es decir, casi duplican la cantidad de matriculados en Universidad pública; solo que algunas de las 23 privadas tienen más de una instalación a nivel nacional, lo que les da más infraestructura.

De ese primer problema, el de la capacidad de infraestructura, surge un segundo, visualizado en la necesidad de regular y controlar el acceso de los de nuevo ingreso, esto., para evitar que el sistema colapse, pues no tiene los mecanismos necesarios como para garantizar la educación a más personas de su capacidad administrativa, docente e infraestructura. Esto es solucionado en gran medida por el examen de admisión que deben aprobar los que quieren ingresar.
Una tendencia para validar el examen de admisión es la de que, debido a la poca infraestructura de las universidades, la demanda excede en gran medida la oferta, lo que obliga a hacer un proceso de selectividad a través de ese examen, donde todos tienen una igualdad de posibilidades (sabiendo que la igualdad es algo no muy posible de realizarla) de ser seleccionados y permitirles el acceso a estudios superiores; todo eso con el fin de no sobresaturar y evitar la mala calidad educativa. Este planteamiento solo muestra la ineficiencia del Estado de garantizar los establecimientos educativos (Art. 53 de la Constitución de la República) de buena calidad para que todos puedan recibir educación. Los que pagan dicha ineficiencia no conforman el Estado, sino aquellos que quedan fuera de la selectividad.

Que la demanda excede a la oferta puede observarse en los datos siguientes. Para el ingreso del 2019, fueron registrados como aspirantes 23,305 estudiantes, de los cuales solo quedaron seleccionados 12,003 (UAIP, 2019). Esto significa que dicho examen inicial de admisión (general y específico) dejó fuera cerca del 51. $5 \%$ del total de registrados, quienes automáticamente ya no tuvieron oportunidad de ingresar. Esto denota la cantidad grande de personas que cada año quieren ingresar a estudios superiores; sin embargo, pocos quedan finalmente admitidos.

El patrón se repite con cierta regularidad en los años anteriores. El gráfico siguiente muestra dichos datos desde el 2014. 


\section{Gráfica 2: Datos de aspirantes y seleccionados por año. Se observa comparación de datos de los últimos cuatro años en cuanto a número de aspirantes y seleccionados en la UES.}

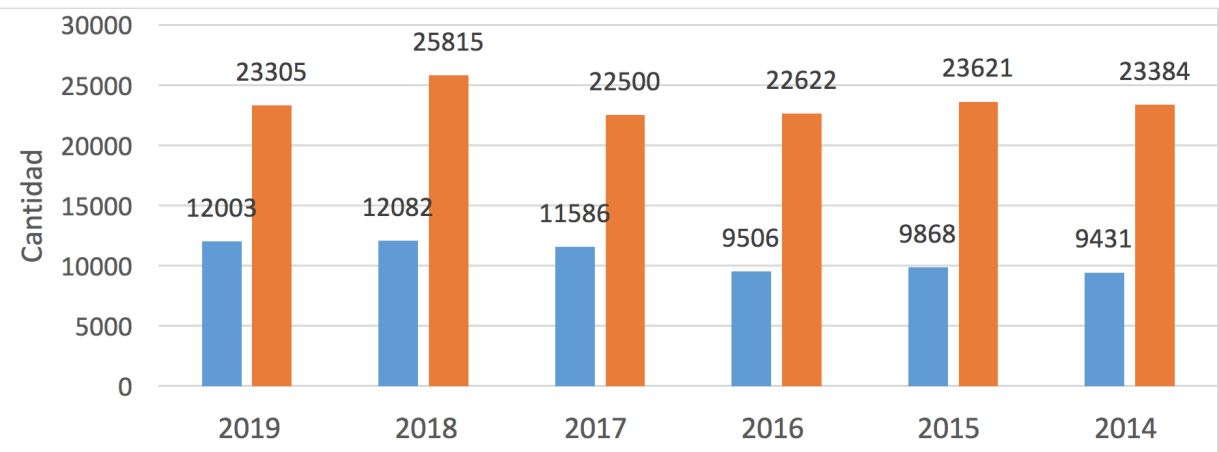

- Seleccionados aspirantes

Fuente: Creación propia a partir de datos de los años 2014 a 2016, extraídos de boletines de la Universidad de El Salvador (2017), y de los años 2017 al 2019 con información de Unidad de Acceso a la Información Pública (UAIP, 2019).

El gráfico 2 muestra datos comparativos en cuanto a la cantidad de admitidos cada año, desde el 2014. Nótese un aumento leve de más de 1000 estudiantes a partir del año 2017 , en contraste a con los tres años anteriores. Si embargo, lo relevante está es la cantidad significativa de aspirantes que no logran ganar un cupo dentro de la Universidad, es decir, no logran obtener el acceso a estudios superiores en la Universidad pública, el cual ronda, en los últimos cuatro años, en más del $50 \%$.

Lo anterior debe ser un dato preocupante, dado que todo ciudadano debe tener derecho a la educación; pero al menos en la Universidad pública no es un hecho que se dé en su totalidad. Desde luego, las causas pueden ser muchas: desde la falta de presupuesto de acuerdo a las exigencias educativas e infraestructurales que requiere la universidad, hasta el poco interés de políticas educativas que tomen en serio el fortalecimiento de la educación superior.

\section{Esbozo desde lo jurídico}

Los derechos humanos están orientados, al menos teóricamente, a lograr el desarrollo de la cultura y la plenitud humana (UNESCO, 2008). Por lo anterior es que la convención sobre los derechos humanos ha sido suscrita por diversos países, lo cual se expresa en la Constitución que rige la nación de cada uno de ellos; esto significa que las expresiones de los derechos humanos, eminentemente universales, están explícitas e implícitas en máxima expresión en las le- 
yes jurídicas de una nación, y es allí donde se debe encontrar la fundamentación de todos los derechos que posee un ciudadano.

Lo anterior es fundamental saberlo, ya que permite mostrar las bases, al menos jurídicas $^{2}$, del tema que ocupa este artículo. Para verificar si los ciudadanos tienen el derecho a recibir educación superior, suponiendo que filosóficamente lo tienen, es menester encontrar la fuente legal que establezca el derecho a la educación en todas sus dimensiones.

La Constitución de la República de El Salvador, en su Artículo 3 establece que "Todas las personas son iguales ante la ley. Para el goce de los derechos civiles no podrán establecerse restricciones que se basen en diferencias de nacionalidad, raza, sexo o religión" (El Salvador, Asamblea Legislativa, 2015, pág. 2); esto implica que en cuanto a la aplicación de la ley todas las personas son iguales, lo que significa que los derechos especificados en dicha Constitución aplican por igual a todas las personas. Salta a la vista el poder de dicho enunciado: si hay una ley que especifique el derecho a la educación superior de toda persona, es aplicable para todas sin distinción alguna, inclusive, aunque se corra el riesgo de salirse de lo jurídico, de las distinciones en cuanto a las ap- titudes que pueda poseer para optar a una educación superior.

Más adelante, específicamente en la sección tercera del Capítulo II, se establece en el Artículo 53 el derecho a la educación y a la cultura, lo cual "es inherente a la persona humana; en consecuencia, es obligación y finalidad primordial del Estado su conservación, fomento y difusión" (El Salvador, Asamblea Legislativa, 2015, pág. 12); el enunciado clave de este Artículo consiste en la manifestación de que ese derecho a la educación es inherente a la persona humana, lo cual supone que el concepto de educación engloba a cualquier persona independientemente de su edad ${ }^{3}$, asimismo de cualquier nivel educativo o tipo de educación que pueda recibir. En pocas palabras, no hay un enunciado que niegue el derecho, y con ello el acceso, a la educación.

No obstante, la base legal no se agota allí. El Artículo 58 deja muy claro cuáles son las distinciones que se deben evitar, es decir, que no deben ser una barrera para cumplir el derecho de recibir la educación en establecimientos educativos (nótese que ya menciona establecimientos educativos), entre los cuales están las distinciones raciales, religiosas, sociales y políticas, y que se puede ver replicado en el Art. 44 de la Ley Orgá-

2 La base filosófica es la propia convención de los derechos humanos. Es filosófica debido a que es producto de un sistema de pensamiento; esto especifica que por el simple hecho de ser seres humanos tenemos derechos que nos son inherentes de manera universal

3 La categoría de la edad es necesario aclararla: el Art. 35 menciona "[el Estado] garantizará el derecho de éstos [menores de edad] a la educación" (El Salvador, Asamblea legislativa, 2015, pág. 7). El Art. 56 ya lo delimita muy puntualmente: parvularia y básica, dejando con mucha claridad que en este caso únicamente es garantizado a menores de edad (ver LEPINA, Art. 81); por el contrario, el Art. 53 lo menciona sin distinción de edad, al menos así se entiende, lo que implicaría abarcar la educación superior. 
nica de la Universidad de El Salvador; esto implica que la persona no tiene ninguna restricción en su derecho de recibir educación universitaria, al contrario, tiene una base legal en la cual fundamentar dicho derecho.

Una vez visto el marco legal que debe garantizar el derecho a la educación, y dentro de ella a la educación superior, conviene hacer la siguiente pregunta: ¿Por qué en la realidad no se cumple ese derecho? Las repuestas varían. Una primera respuesta radica en que realmente todos tienen la oportunidad de recibir educación superior. Salvo una condición: pasar el examen de admisión establecido, en el caso de la Universidad de El Salvador, en el reglamento general del proceso de ingreso; caso contrario, queda fuera del acceso a la educación superior, destruyendo por sí mismo el derecho a la educación superior y demostrando que no hay igualdad en el acceso a dicha instancia.

En efecto, la Ley Orgánica de la Universidad de El Salvador (2010) en el Artículo 43 expresa que el ingreso a la UES estará regulado por una ley especial. El Reglamento de la Ley Orgánica de la Universidad de El Salvador (2017) en su Art. 67 especifica cuáles son los requisitos para el ingreso, donde uno de ellos es haber obtenido el puntaje requerido en las pruebas de admisión. Por otro lado, el Reglamento General del Proceso de Ingreso de aspirantes a estudiar en la Universidad de El Salvador (2005) también reitera el requisito de obtener el rendimiento mínimo en el examen de admisión para ser seleccionado.
La normativa para el acceso a la educación superior pública está regulada, lo que implica que es un proceso organizado, sistematizado y con base jurídica. Sin embargo, su planteamiento entra en contradicción con el derecho a la educación que defiende la Constitución de la República. Por lo tanto, es necesario debatir este dilema, puesto que no se le puede negar a ninguna persona la oportunidad de formarse profesionalmente.

\section{Los programas y las políticas de educación frente al acceso a la educa- ción superior}

Hay que resaltar que la educación superior es un eslabón que históricamente no ha sido tomado con seriedad dentro de los planes educativos de la nación. Esbozando los programas de los últimos 20 años se tiene lo siguiente: a) La reforma educativa de 1995, que se enfocó en los cambios administrativos y a nivel de cobertura (El Salvador, Ministerio de Educación, MINED, 1995). b) El Plan Nacional de Educación 2021 (MINED, 2005), que establece políticas para lograr la escolaridad básica que llega hasta bachillerato, no considerando dentro la educación superior, aun cuando apuesta fuertemente a la formación tecnológica y técnica; c) Asimismo, plantea en su Línea estratégica 1 el acceso a la educación, e igualmente la reduce hasta la educación media. d) Con el Plan Social Educativo 2009-2014 "Vamos a la escuela" (MINED, 2009), el acceso lo menciona hasta educación media. e) En cuanto al fortalecimiento a la educación superior (Línea estratégica $G$ ) se orienta a la 
investigación, la creación de convenios nacionales e internacionales y la revisión del marco legal, entre otros, no detallando ninguna acción que tome en cuenta la ampliación de infraestructura.

Por otro lado, el documento del Plan Quinquenal de Desarrollo (2014-2019), en el objetivo 2, se enfoca en la mejora de la cobertura y la infraestructura de la educación; no obstante, la educación superior queda prácticamente fuera de vista, pues solo es mencionada fugazmente en la Línea estratégica E.2.2, L.2.4.6, que literalmente dice así: "Fortalecer la cobertura y calidad de la educación superior, articulándola con los otros niveles del sistema educativo y fomentando la investigación y generación de conocimientos" (El Salvador, Secretaría Técnica y de Planificación, 2015, pág. 120).

El énfasis ha estado, en gran medida, orientado a la cobertura y la calidad de la educación básica y media, aquella que es la mínima que toda persona salvadoreña debe poseer; por lo tanto, las políticas educativas se dirigen a satisfacer problemas como deserción escolar, baja matrícula, matrícula, reprobación y sobreedad, entre otros.

Quizá sea eso lo que limita tomar en seriedad la educación superior pública, en la medida en que el Estado busca superar las tasas de analfabetismo a partir de políticas y proyectos en educación básica, sobre todo, y educación media. Esto justificaría, en gran medida, el poco interés en el fortalecimiento de la educación superior.
En cuanto al presupuesto para el 2017 se le asignó a educación superior el $1.5 \%$ del presupuesto general de la nación (periódico "El Universitario, 2017). Entre los acuerdos están el incremento anual de al menos 26 millones de dólares, aumentando $0.5 \%$ cada año para alcanzar el $3 \%$ en el año 2019 ("El universitario", 2016). Esto es un avance significativo, aunque no suficiente, debido a que el $3 \%$ sigue siendo un porcentaje bajo en comparación con las exigencias de la educación superior. No obstante, se debe esperar que esto sea el inicio de los esfuerzos por considerar a la educación superior pública, como una instancia de primer orden a nivel de sociedad.

Aun con todas las limitantes presupuestarias y de recursos, hay intentos de tomar en cuenta a todos los aspirantes (ejemplo de esto es la Universidad de El Salvador, FMOcc, con sus procesos de ampliación de cupos); pero la infraestructura, los recursos y el financiamiento se quedan cortos como para darles esa posibilidad a todos. En esta perspectiva, voluntad existe por parte de las autoridades y movimientos estudiantiles; pero las condiciones económicas la limitan y fuerzan a crear mecanismos de selección de ingreso.

\section{El papel del estatus socioeconómi- co en el acceso a educación superior}

La educación siempre ha estado ligada a cuestiones de estatus social y económicas. Desde los griegos, acceder a la educación era una oportunidad exclusiva de los altos estratos de la sociedad, pues solo ellos tenían las condiciones para costearla en tér- 
minos económicos (López, 2015). Esta pauta elitista de la educación ha seguido hasta nuestros días, con el único cambio de que, al menos en El Salvador, la educación pública ha mejorado la cobertura hacia las clases pobres y de bajo estatus.

En la actualidad, desde la perspectiva socioeconómica, esa posibilidad económica no es tan grande, pues en El Salvador el 29.2 $\%$ es de pobreza (6.2 extrema y 23.0 relativa) según datos de EHPM del 2018, o sea que hay una cantidad significativa de hogares que no poseen ni el mínimo necesario para costear la canasta básica alimentaria (CBA), lo que significa para muchos jóvenes que la única posibilidad de estudiar en la Universidad es en la pública, pues para una privada difícilmente puedan costearla.

Por otro lado, hay que tener en cuenta que el estatus social juega un papel importante en la educación superior. Al respecto, en un Editorial de Radio YSUCA (2010) se expresa lo siguiente:

"La educación universitaria es una de las principales vías de ascenso social en la medida que constituye puerta de entrada a muchas oportunidades. Hay una clara diferencia entre ser graduado universitario o no serlo: en el mercado laboral, los salarios de los que poseen un título universitario son mayores a los de aquellos que no cuentan con estudios superiores" (párrafo 4).

Es indiscutible que el título universitario mejora la situación social y económica de quien lo ostenta. En efecto, los empleos me- jor remunerados piden entre sus requisitos poseer un título universitario. Ligado a esto, está la creciente demanda de cupos en la Universidad pública. La titulación universitaria también implica mejora de ingresos salariales, en contraste con aquellos que no la poseen. Desde aquí adquiere sentido la alta cantidad de aspirantes que desean entrar a la Universidad, por lo que es obligación del Estado crear las condiciones para garantizar la educación superior.

El dilema surge cuando se restringe el acceso. Muchos de los aspirantes que no logran cupo no tienen las condiciones necesarias como para pagar estudios en universidades privadas. Y agreguemos que, en cuanto a la formación intelectual, hay unos estudiantes más dotados que otros, y ello está ligado a condiciones socioculturales y económicas de cada uno, lo que significa que aquellos que tienen familias con buen índice de ingreso económico tienen más posibilidades de acceder a la educación superior.

Conviene detenerse en lo escribo arriba. F. Gutiérrez (1985), por ejemplo, en su libro "Educación como praxis política, planteándose también este mismo dilema, llega a concluir lo siguiente:

"Los estudiantes hijos de campesinos, de artesanos y obreros están económicamente condicionados de tal manera que de poco les sirve el precepto legal que asegura que todo habitante de la república tiene derecho a recibir una educación amplia, y de poco le aprovecha que el Estado le garantice la igualdad de oportunidades. 
Confundir igualdad de oportunidades con igualdad de posibilidades es una manifestación ideológica más" (página. 33).

Esto conlleva a asociar un examen de admisión como un elemento ideológico y político, ya que "toda selección implica necesariamente exclusión" (F. Gutiérrez, ob. cit. 1985, pág. 35) y lleva necesariamente una discriminación social y socioeconómica. En ese sentido, "el uso excesivo de pruebas estandarizadas conlleva un obstáculo para los jóvenes de bajo perfil socioeconómico" (como escriben Apple M. y Beane W, en su libro "Escuelas democráticas", 2000); así ya no es una igualdad de oportunidades, sino que una igualdad de posibilidades, donde sale ganador aquel que posee mejores condiciones socioeconómicas, enterrando de una sola palada el derecho a la educación.

Este supuesto social y económico también se da en la educación superior pública: la universidad debe tener los mejores estudiantes, supuesto que implica también discriminación social y económica de las que hablan F. Gutiérrez (ob. cit.1985) y Apple M. y Beane W. (ob. cit., 2000), y que de paso vuelve complicado el derecho a la educación en todos sus niveles, ya que los mejores estudiantes son aquellos que han tenido disponibilidad de recursos para poseer un mejor caudal de conocimientos. Si bien es cierto que obtener el puntaje necesario por suerte es una posibilidad, no es lo que busca la selectividad en sí.

Esta discriminación económica y social también está referida de manera implícita a las políticas del mercado. En esta dimensión, el control de ingreso a la Universidad está regido por los estándares de calidad que pide el mercado, ya que, como lo expresa Lundgren (1992), se prepara a profesionales para que se desempeñen en las diferentes áreas del mercado. No se forma, entonces, para tener profesionales intelectuales y prácticos, capaces de enfrentarse a los grandes problemas de la sociedad para transformarlo.

El acceso a la educación superior está intrincado en muchas cuestiones políticas, sociales, económicas y jurídicas, por lo que no se puede resolver tan fácilmente la problemática. Su discusión debe ser crítica, considerando tanto sus causas y consecuencias directas e indirectas. Desde luego, esto es posible en la medida de aceptar primero que la educación superior no debe ser un privilegio de unos pocos, más bien un derecho de todos.

\section{Algunas alternativas para el acceso a la educación superior pública}

La Universidad de El Salvador ha estado constantemente asumiendo nuevos retos y realizando mejoras para mejorar la cobertura y la calidad de la educación. En ese sentido, hay varios proyectos que ya se han puesto en marcha, y otros se pondrán a largo plazo, los que favorecerán a que la UES se convierta en una institución educativa más abierta a la población estudiantil.

\subsection{Universidad en línea}

Uno de los proyectos más innovadores y que lanza a la Universidad a la vanguardia de las 
nuevas formas de educación, es el de la Universidad en línea-educación a distancia. Esta modalidad inició en el 2017 y actualmente cuenta con ocho carreras en su oferta académica (Universidad de El Salvador, 2017).

Este proyecto da posibilidades de estudio a aquellos que tienen compromisos de trabajo o viven en lugares demasiado alejados al centro de estudios universitarios, lo que les obstaculiza realizar estudios superiores; sin embargo, con esta modalidad pueden obtener algunas facilidades, ya que solo reciben clases presenciales cada determinado tiempo.

Si bien es cierto que en esta modalidad también está presente el examen de admisión, siempre es un proyecto que ofrece oportunidad de estudios superiores a una minoría de bachilleres con condiciones adversas y económicas, con miras a que obtengan una formación que les ayude a superarse.

\subsection{Excepción de examen de admisión para bachilleres}

Una de las medidas administrativas muy loables para el acceso a la educación superior pública es la eliminación del examen de admisión para los bachilleres que pertenecen a municipios de extrema pobreza. Con esta medida se busca que jóvenes que viven en alguno de los 91 municipios catalogados como de extrema pobreza puedan estudiar una carrera universitaria (elsalvador.com, 2017).

Esta política de inclusividad ayuda a aumentar la cobertura y eliminar las barreras de desigualdad social para el derecho a la educación. Además, estos estudiantes pueden optar a cualquiera de las becas que la universidad ofrece a través de la Unidad de estudios socioeconómicos.

\subsection{Ampliación de sedes regionales}

Una de las soluciones más ambiciosas a la falta de infraestructura amplia es la creación de nuevas sedes regionales de la UES, aparte de las cuatro con las que cuenta en la actualidad, lo que favorecería en aumentar la capacidad de admisión de estudiantes, al tiempo que bajaría la sobresaturación en las otras sedes.

Uno de los intentos más recientes es la iniciativa de crear una sede en el departamento de Ahuachapán. Este anhelo se ha visto cumplido a partir del 2019, cuando la sede de Koika, en Ahuachapán, ha sido prestada para ofertar carreras del plan especial para profesores, la maestría en docencia superior, curso de inglés intensivo y algunos diplomados. Esta apertura de nueva sede es un gran logro de las autoridades de la FMOcc en su intento por democratizar la educación superior.

En Sonsonate es donde se ha dado la iniciativa más reciente y concretizada de abrir nueva sede. Este intento ha sido gestionado por las autoridades de la Facultad Multidisciplinaria de Occidente, Dr. Raúl Azcúnaga e Ing. Roberto Sigüenza, quienes en colaboración con el alcalde de Sonzacate, Héctor Orellana, han planificado un terreno en co- 
modato para construir la sede, que se planee esté lista para el año 2020 (Salguero, 2018).

\section{A modo de conclusión}

Adquirir un título universitario ${ }^{4}$ es una exigencia de primer orden para desempeñarse en el mundo laboral; por lo tanto, la educación superior debe ser una prioridad del Estado salvadoreño, pues es necesario que este garantice la formación educativa de los salvadoreños hasta la educación superior, dado que ello asegura la promoción de profesionales acordes a la nueva realidad del mundo globalizado y cada vez más exigente en cuanto al conocimiento.

El examen de admisión no es un mecanismo que garantice esa oportunidad de formación profesional; al contrario, es una medida excluyente. En realidad, una alternativa de solución radica en la ampliación del personal docente, administrativo y, sobre todo, de la infraestructura. Lógicamente, para llevar a cabo eso se requiere de un aumento presupuestario considerable, destinado a la edu- cación superior, que permita la ampliación de cobertura; pero también que permita las condiciones mínimas para desarrollarla. Esto dará oportunidad a muchos jóvenes de cumplir su sueño de estudiar una carrera universitaria y superarse académicamente.

Lo que se busca, en primera instancia, no es más que el cumplimiento constitucional de la igualdad y de la garantía del acceso a una educación completa de los salvadoreños, debido a que el futuro depende en gran medida de los profesionales que forma nuestra alma máter.

Se deben buscar alternativas que sigan ampliando la posibilidad de garantizar la educación superior a toda la comunidad educativa apta para estudiarla, pues ello es un derecho $y$, a la vez, una necesidad para fortalecer la educación de los ciudadanos.

En fin, la apuesta por el fortalecimiento de la universidad pública debe ser una prioridad, pues en ella radica el progreso, y sin ella se produce estancamiento de la cultura salvadoreña.

4 Ahora se necesita más que una licenciatura; tener una maestría ya es necesario para competir con la oferta laboral. 


\section{Referencias bibliográficas}

Apple, M. (2001). “Educar como Dios manda”. Buenos Aires: Paidós.

Apple, M. y Beane, W., (2000). "Escuelas democráticas". Madrid: Morata.

Elsalvador.com. (agosto, 2017). "Los jóvenes que vivan en estos municipios no harán examen de admisión en la UES". Disponible en http://www.elsalvador.com/ noticias/nacional/365342/los-jovenes-que-vivan-enestos-municipios-no-haran-examen-de-admisionen-la-ues/

El Salvador, Asamblea Legislativa (2016). "Constitución de la República". Disponible en http://www.asamblea.gob. sv/eparlamento/indice-legislativo/buscador-de-documentos-legislativos/constitucion-de-la-republica

El Salvador, Corte Suprema de Justicia. (2011). "Ley de protección integral a la niñez y adolescencia”. San Salvador, El Salvador: Comisión coordinadora del sector de justicia.

"El Universitario". (2016). "Ejecutivo apoyará propuesta de incremento al 3\% para el presupuesto de la Universidad de El Salvador". Disponible en http://www.eluniversitario.ues.edu.sv/5034-2016-09-09-14-01-07

"El Universitario". (Agosto-2017). "3,557 estudiantes aprueban examen General de Conocimiento para ingresar a la UES en el 2017". Disponible en http://www.eluniversitario.ues.edu.sv/5191-2016-11-04-20-51-15

Gutiérrez, F., (1985). "Educación como praxis política". México D.F.: Siglo XXI

López, D. E. (2015). "La construcción histórica de la Educación superior. Modelos universitarios y docentes". San Salvador: Centro de Investigaciones en Ciencias y Humanidades. Universidad Dr. José Matías Delgado.

Lundgren, U. (1992). "Teoría del currículum y escolarización". Madrid: Morata.

Ministerio de Economía. (2017). “Encuesta de hogares con propósitos múltiples (EHPM)". San Salvador: autor

Ministerio de Educación. (1995). "Reforma educativa en marcha. Documento III: Lineamientos generales del plan decenal 1995-2005". San Salvador; autor:
Ministerio de Educación. (2005). "Plan nacional de educación 2021. Metas y políticas para construir el país que queremos". San Salvador; autor:

Ministerio de Educación. (2009). "Programa social educativo Vamos a la escuela". 2009-2014. San Salvador; autor:

Ministerio de Educación. (2015). "Resultados de información estadística de Instituciones de Educación superior 2014". San Salvador; autor:

Ministerio de Educación. (2016). "Resultados de información estadística de Instituciones de Educación superior 2015". San Salvador; autor:

Ministerio de Educación. (2018a). "Resultados de información estadística de Instituciones de Educación superior 2016". San Salvador; autor: (académicos, 2019).

Ministerio de Educación. (2018b). "Resultados de información estadística de Instituciones de Educación superior 2017". San Salvador: Editorial e impresos Panamericana

Radio YSUCA. (2010). Editorial "El limitado acceso a la educación superior". Disponible en: http://www.uca.edu. sv/noticias/texto-1060

Salguero, M. (2 de agosto de 2018). Edificio UES estaría listo en 2020. "La Prensa Gráfica”. Disponible en https:// www.laprensagrafica.com/elsalvador/Edificio-UESestaria-listo-en-2020-20180801-0095.html

Secretaría de Asuntos Académicos. (2019). “Estadístico de estudiantes inscritos por facultad al año". Disponible en https://saa.ues.edu.sv/nosotros/estadistica/completo

Secretaría técnica y de planificación. (2015). "Plan quinquenal de desarrollo 2014-2019". San Salvador; autor:

Unidad de acceso a la información pública, UAIP. (2019). "Datos estadísticos de nuevo ingreso Universidad de El Salvador".

Unesco. (2018). "Declaración universal de los derechos humanos". Disponible en http://unesdoc.unesco.org/ images/0017/001790/179018m.pdf

Universidad de El Salvador (2017). "Universidad en línea". Disponible en http://distancia.ues.edu.sv/\#sedes 
Universidad de El Salvador. (2005).“Reglamento general de proceso de ingreso de aspirante a estudiar en la Universidad de El Salvador". Disponible en http://www. transparencia.ues.edu.sv/sites/default/files/PDF/Reglamento\%20General\%20de\%20Proceso\%20de $\% 20$ Ingreso\%20(versión\%20divulgativa).pdf

Universidad de El Salvador. (2010). "Ley orgánica de la Universidad de El Salvador". Disponible en http://www. ues.edu.sv/descargas/ley_organica_UES.pdf

Universidad de El Salvador. (2013). "Legislación universitaria. Libro segundo. Recopilación académica". San Salvador.

Universidad de El Salvador. (2017). "Boletín estadístico. Primer ingreso 2014". Disponible en: https://expediente4.ues.edu.sv/uiu/2014/Bole2014.pdf

Universidad de El Salvador. (2017). “Boletín estadístico. Primer ingreso 2015". Disponible en: https://expediente4.ues.edu.sv/uiu/2015/Bole2015.pdf

Universidad de El Salvador. (2017). “Boletín estadístico. Primer ingreso 2016". Disponible en: https://expediente4.ues.edu.sv/uiu/2015/Bole2016.pdf
Universidad de El Salvador. (Agosto-2017). “Población de estudiantes para el año 2017". Disponible en https:// expediente4.ues.edu.sv/estadisticas/poblacion_estudiantil.php?\&npag=1\&anio=2017

Universidad de El Salvador. (Agosto-2017). "Población de estudiantes para el año 2016". Disponible en https:// expediente4.ues.edu.sv/estadisticas/poblacion_estudiantil.php?\&npag=1\&anio=2016

Universidad de El Salvador. (Agosto-2017). “Población de estudiantes para el año 2015". Disponible en https:// expediente4.ues.edu.sv/estadisticas/poblacion_estudiantil.php?\&npag=1\&anio=2015

Universidad de El Salvador. (Agosto-2017). "Población de estudiantes para el año 2014". Disponible en https:// expediente4.ues.edu.sv/estadisticas/poblacion_estudiantil.php?\&npag=1\&anio=2014 